Global Journal of Pure and Applied Mathematics.

ISSN 0973-1768 Volume 11, Number 3 (2015), pp. 1283-1287

(C) Research India Publications

https://dx.doi.org/10.37622/GJPAM/11.3.2015.1283-1287

\title{
A Result on the Consecutive Adjacent Degree of a Semigraph
}

\author{
S.Saravanan ${ }^{1}$ and R.Poovazhaki ${ }^{2}$ \\ ${ }^{1}$ Department of Mathematics, R.M.D. Engineering College, Kavaraipettai-601 206, \\ Tamilnadu, India. \\ ${ }^{2}$ Principal, E.M.G. Yadava Women's College, Madurai-625 014, Tamilnadu, India.
}

\begin{abstract}
Semigraphs introduced by E.Sampathkumar [3] is a new type of generalization of the concept of graph. A semigraph $G=(V, X)$ on the nonempty set of vertices $V$ and the set of edges $X$ consists of $n$-tuples $\left(u_{1}, u_{2}, \ldots, u_{n}\right)$ of distinct elements belonging to the set $V$ for various $n \geq 2$, with the following conditions:

(i) Any $n$-tuple $\left(u_{1}, u_{2}, \ldots, u_{n}\right)=\left(u_{n}, u_{n-1}, \ldots, u_{1}\right)$ and

(ii) Any two such tuples have at most one element in common.

For a vertex $v$ in a semigraph $G$, we can define various types of degrees. This paper presents a new result on the sum of consecutive adjacent degree in semigraphs.
\end{abstract}

Keywords: Semigraph, $k$-Uniform semigraph, Consecutive adjacent degree, Edge degree.

2010 Mathematics Subject Classification: 05C07, 05C99.

\section{Introduction}

Graphs have many properties that are enjoyed by vertices are not applicable for edges. To rectify these so called defects in graph theory E. Sampathkumar [3] introduced the new structure namely semigraphs. Semigraph is a kind of straightforward generalization of every concept in graphs which may not be possible in a similar structure called Hypergraphs. Because in semigraphs the vertices in each edge are arranged in an order. Thus semigraphs are more closely related to graphs than to hypergraphs. 
All semigraphs considered here are nonempty, finite, and undirected. Let $G=(V, X)$ be a semigraph with $p$ vertices and $q$ edges. We give definitions as in [3].

Definitions 1.1[3].

For a vertex in a semigraph $G$, we define various types of degrees as follows:

Degree: $\operatorname{deg} v$ is the number of edges having $v$ as an end vertex.

Edge Degree: $\operatorname{deg}_{e} v$ is the number of edges containing $v$.

Adjacent Degree: $\operatorname{deg}_{a} v$ is the number of vertices adjacent to $v$.

Consecutive Adjacent Degree: $\operatorname{deg}_{c a} v$ is the number of vertices which are consecutively adjacent to $v$.

Definition 1.2[2].

A semigraph is $k$-uniform if every edge contains exactly $k$-vertices.

Theorem 1.3[3].

Let $G=(V, X)$ be a semigraph where $V=\left(v_{1}, v_{2}, \ldots, v_{p}\right)$ and $X=\left(E_{1}, E_{2}, \ldots, E_{q}\right)$. Then

1. $\sum_{i=1}^{p} \operatorname{deg} v_{i}=2 q$.

2. $\sum_{i=1}^{p} \operatorname{deg}_{e} v_{i}=\sum_{i=1}^{q}\left|E_{i}\right|$.

3. $\sum_{i=1}^{p} \operatorname{deg}_{a} v_{i}+\sum_{i=1}^{p} \operatorname{deg}_{e} v_{i}=\sum_{i=1}^{q}\left|E_{i}\right|^{2}$.

Sampathkumar [3] had proved the above theorem on sum of degrees, sum of edge degrees and sum of adjacent degrees of vertices. This motivates us to find a new result on the sum of consecutive adjacent degrees in a semigraph which is discussed in the next section.

\section{Main Results}

\section{Theorem 2.1}

The sum of consecutive adjacent degrees of points of a semigraph $G$ is the excess of twice the sum of edge degrees of points of $G$ over the sum of degrees of points of $G$. i.e $\sum_{i=1}^{p} \operatorname{deg}_{c a} v_{i}=2 \sum_{i=1}^{p} \operatorname{deg}_{e} v_{i}-\sum_{i=1}^{p} \operatorname{deg} v_{i}$.

\section{Proof}

Let $G$ be a semigraph. We observe that for any vertices $v, \operatorname{deg}_{c a} v=\operatorname{deg}_{e} v+$ number of edges having $v$ as a middle vertex. The number of middle vertices in an edge $E_{i}$ in 
a semigraph $G$ is $\left|E_{i}\right|-2$ and the contribution of these vertices to $\sum_{i=1}^{p} \operatorname{deg}_{c a} v_{i}$ is $\left|E_{i}\right|-2$. Hence we have,

$$
\begin{aligned}
\sum_{i=1}^{p} \operatorname{deg}_{c a} v_{i} & =\sum_{i=1}^{p} \operatorname{deg}_{e} v_{i}+\sum_{i=1}^{q}\left(\left|E_{i}\right|-2\right) . \\
& =\sum_{i=1}^{p} \operatorname{deg}_{e} v_{i}+\sum_{i=1}^{p} \operatorname{deg}_{e} v_{i}-2 q . \\
\sum_{i=1}^{p} \operatorname{deg}_{c a} v_{i} & =2 \sum_{i=1}^{p} \operatorname{deg}_{e} v_{i}-\sum_{i=1}^{p} \operatorname{deg} v_{i} .
\end{aligned}
$$

\section{Example 2.2}

Consider semigraphs given in Figure 1.

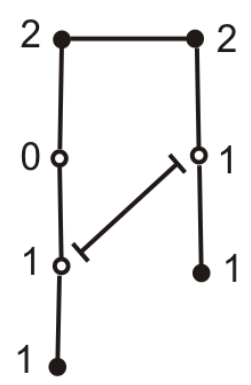

Degrees
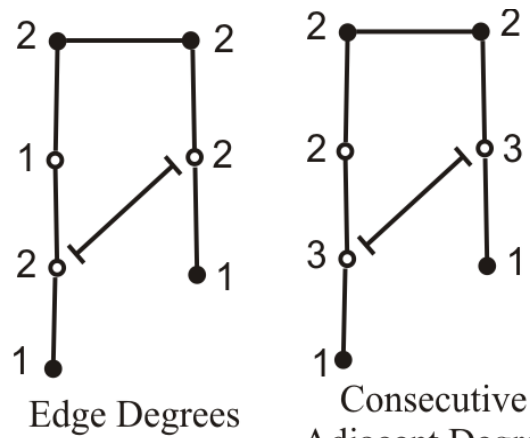

Consecutive

Adjacent Degrees

Figure 1:

The sum of degrees, sum of edge degrees and sum of consecutive adjacent degrees of semigraphs given in Figure 1 are 8,11 and 14 respectively. Hence the result given in Theorem 2.1 is satisfied.

\section{Corollary 2.3}

If $G=(V, X)$ is a semigraph, then $\sum_{i=1}^{p} \operatorname{deg}_{c a} v_{i}+\sum_{i=1}^{p} \operatorname{deg} v_{i}=2 \sum_{i=1}^{q}\left|E_{i}\right|$.

\section{Proof}

By above theorem, $\sum_{i=1}^{p} \operatorname{deg}_{c a} v_{i}+\sum_{i=1}^{p} \operatorname{deg} v_{i}=2 \sum_{i=1}^{p} \operatorname{deg}_{e} v_{i}$.

$$
=2 \sum_{i=1}^{q}\left|E_{i}\right| \text {. }
$$




\section{Corollary 2.4}

Let $G=(V, X)$ be a semigraph where $V=\left(v_{1}, v_{2}, \ldots, v_{p}\right)$ and $X=\left(E_{1}, E_{2}, \ldots, E_{q}\right)$. Then $\sum_{i=1}^{p} \operatorname{deg}_{c a} v_{i}=2\left[\sum_{i=1}^{q}\left|E_{i}\right|-q\right]$.

\section{Proof}

By Theorem 2.1, $\sum_{i=1}^{p} \operatorname{deg}_{c a} v_{i}=2 \sum_{i=1}^{p} \operatorname{deg}_{e} v_{i}-\sum_{i=1}^{p} \operatorname{deg} v_{i}$.

$$
=2\left[\sum_{i=1}^{q}\left|E_{i}\right|-q\right] \text {. }
$$

Theorem 2.5[1].

In any semigraph, the number of vertices of odd consecutive adjacent degree is even.

\section{Proof}

Let $v_{1}, v_{2}, \ldots, v_{n}$ denote the vertices of odd consecutive adjacent degree and $w_{1}, w_{2}, \ldots, w_{m}$ denote the vertices of even consecutive adjacent degree in $G$. By Corollary $2.4, \sum_{i=1}^{n} \operatorname{deg}_{c a} v_{i}+\sum_{i=1}^{m} \operatorname{deg}_{c a} w_{i}$ which is even. Further $\sum_{i=1}^{m} \operatorname{deg}_{c a} w_{i}$ is even.

Hence $\sum_{i=1}^{n} \operatorname{deg}_{c a} v_{i}$ is also even. But $\operatorname{deg}_{c a} v_{i}$ is odd for each $i$. Hence $n$ must be even.

\section{Example 2.6}

If $G$ is a $k$-uniform semigraph with pvertices and qedges, then $\sum_{i=1}^{p} \operatorname{deg}_{c a} v_{i}=2 q(k-1)$.

\section{Solution}

Since $G$ is $k$-uniform semigraph, $\sum_{i=1}^{q}\left|E_{i}\right|=q k$.

By Corollary 2.4, $\sum_{i=1}^{p} \operatorname{deg}_{c a} v_{i}=2\left[\sum_{i=1}^{q}\left|E_{i}\right|-q\right]$.

$$
=2 q(k-1) \text {. }
$$

Example 2.7[1].

Let $G$ be a $k$-uniform semigraph all of whose vertices have consecutive adjacent degree $\ell$ or $\ell+1$. If $G$ has $n>0$ vertices of consecutive adjacent degree $\ell$, then $n=p(\ell+1)-2 q(k-1)$. 


\section{Solution}

Since $G$ is $k$-uniform semigraph, $\sum_{i=1}^{q}\left|E_{i}\right|=q k$. Since $G$ has $n$ vertices of consecutive adjacent degree $\ell$, the remaining $(p-n)$ vertices have consecutive adjacent degree $\ell+1$. Hence $\sum_{v \in V} \operatorname{deg}_{c a} v=n \ell+(p-n)(\ell+1)$.

$$
\begin{aligned}
& 2 q(k-1)=p(\ell+1)-n . \\
& n=p(\ell+1)-2 q(k-1) .
\end{aligned}
$$

\section{Example 2.8}

Consider the 3-uniform semigraph given in Figure 2 with $p=5, q=2, k=3, \ell=1$ and $n=2$.

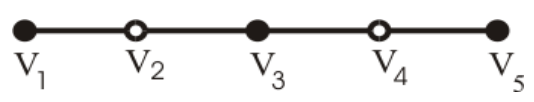

Figure 2: 3-Uniform Semigraph

$p(\ell+1)-2 q(k-1)=10-8=2$. The result given in equation (1) is satisfied.

\section{References}

[1] Frank Harary, Graph Theory, Narosa Publishing House, 1988.

[2] S.Gomathi, R.Sundareswaran, V.Swaminathan, $(m, e)-$ domination in Semigraphs, Electronics notes in Discrete Mathematics, 33 (2009) 75-80.

[3] E.Sampathkumar, Semigraphs and their applications, Report on the DST Project, May 2000. 
